Research Paper

\title{
High Reproducibility of Adhesion Formation in Rat with Meso-Stitch Approximation of Injured Cecum and Abdominal Wall
}

\author{
Daniel Poehnert ${ }^{\circledR}$, Mahmoud Abbas², Hans-Heinrich Kreipe ${ }^{2}$, Juergen Klempnauer ${ }^{1}$, Markus Winny ${ }^{1}$ \\ 1. Department of General, Visceral and Transplantation Surgery, Hannover Medical School (MHH), Hannover, Germany \\ 2. Department of Pathology, Hannover Medical School (MHH), Hannover, Germany
}

$\bowtie$ Corresponding author: Dr. Daniel Poehnert, PhD. Department of General, Visceral and Transplantation Surgery, Hannover Medical School (MHH), Carl-Neuberg-Strasse 1, 30625 Hannover, Germany. Email: poehnert.daniel@mh-hannover.de; Phone: +495115326534; Fax: $+495115324010$

() Ivyspring International Publisher. This is an open-access article distributed under the terms of the Creative Commons License (http://creativecommons.org/ licenses/by-nc-nd/3.0/). Reproduction is permitted for personal, noncommercial use, provided that the article is in whole, unmodified, and properly cited.

Received: 2014.02.18; Accepted: 2014.11.02; Published: 2015.01.01

\begin{abstract}
Objective: Peritoneal adhesions following surgery are a common, serious pathology with severe complications. Appropriate animal adhesion models are essential for the assessment of adhesion preventing medical devices. This study introduces a variation of an established rat model in which highest degree adhesions are induced with excellent reproducibility (OPAM = optimized peritoneal adhesion model). Thus, this model seems to be eligible to study effects of adhesion preventing devices.

Methods: 24 Lewis male rats were divided into four groups (OPAM, WSFX, sham-OPAM, sham-WSFX). The OPAM technique comprised cecal abrasion, creation of an abdominal wall defect plus approximation of injured areas by a suture, which was compared to a setting of lesions without suture fixation (WSFX). All rats were sacrificed at day 7. Macroscopic and histopathological evaluations were performed. Results were statistically analyzed using ANOVA and Dunnett's test.

Results: In OPAM rats macroscopic analyses revealed a $90 \%$ incidence adhesion of cecum to the abdominal wall, all adhesions imposing as complete agglutination. In WSFX animals incidence of adhesions formation was $75 \%$, while in both sham groups there were no adhesions at all. Histology showed the structure of adhesions with merged smooth muscle of colon and skeletal muscle of abdominal wall in all cases.

Conclusion: OPAM technique provides adhesions of injured areas with a better probability than with conventional methods. All OPAM adhesions impressed as highest degree adhesions, i.e. agglutination. Due to high reproducibility in incidence and extend of adhesion formation, the OPAM is recommended for testing of adhesion prevention medical devices.
\end{abstract}

Key words: Adhesion, Prevention, Rat, Cecal Abrasion, OPAM.

\section{Introduction}

Adhesions are considered to be a substantial medical problem ${ }^{1,2}$. They can cause chronic pain and female secondary infertility leading to severely reduced quality of life ${ }^{3-5}$. Furthermore, adhesions are the most frequent reason for intestinal obstruction associated with a $4.3-13 \%$ rate of mortality ${ }^{5}$. There is general agreement that the majority of adhesions result from surgical trauma to the peritoneum. Adhesions cause a considerable burden to the health system ${ }^{6}$. Billions of dollars are spent annually to treat the 
complications of adhesions 7,8. Despite decades of research, no approach to reduce the formation of adhesions has been wholly satisfactory ${ }^{9}$.

Many research groups have used adhesion models including cecal abrasion and/or abdominal sidewall defect, but results have been inconsistent 6,10-13. In 1995 Harris et al. introduced a model mimicking a clinical situation with parietal and visceral peritoneal lesion to induce adhesion formation for comparison of measures for adhesion prevention ${ }^{14}$. Hoffmann et al. (2009) induced adhesions in the same way, but introduced a more detailed scoring system to allow better differentiation 15 .

Our study establishes a variant of the Harris group adhesion model increasing the incidence and intensity of adhesion formation. The use of our stringent model can be a basis for a better comparability in future animal studies.

\section{Materials and Methods}

All protocols were conducted in accordance with animal protection laws and were approved by the local authority. A total of 24 male Lewis rats weighing 270-361 g (mean $329 \mathrm{~g} \pm 26 \mathrm{~g}$ ) were used for this study. All animal experiments were performed at Zentrales Tierlabor, Hanover Medical School, Germany, providing the knowledge (housing, caretaking, etc.) to assure life quality of laboratory animals.

\section{Preoperative Preparation}

All animals were fed a commercial diet ad libi-
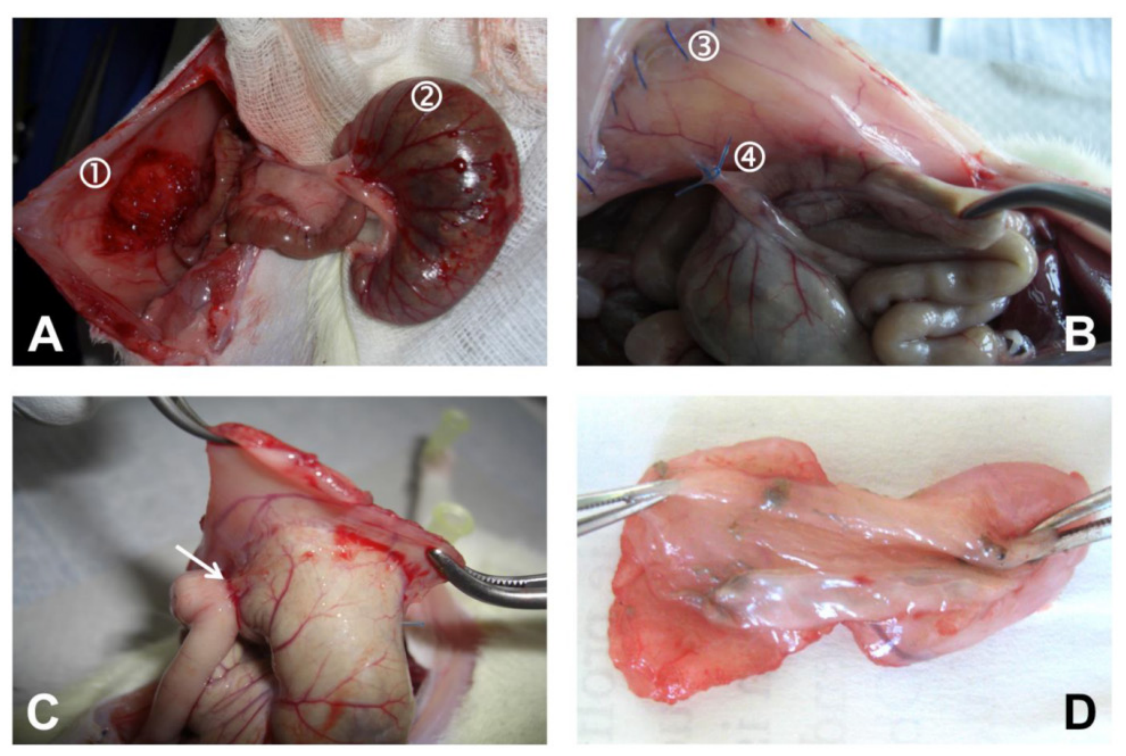

Figure 1: Representative photographic images of rat cecum and abdominal wall taken during operation and autopsy. (A) Operative situs after creation of abdominal wall defect (1) and abrasion of cecum (2). (B) Situs upon autopsy with $4 / 0$ Prolene ${ }^{\circledR}$ running suture for closure of longitudinal laparotomy incision (3) and another 4/0 Prolene ${ }^{\circledR}$ suture for approximation of cecum and abdominal wall (4), note the severe agglutination (highest scores) of abdominal wall and cecum in an animal undergoing OPAM after one week. (C) Area of agglutination excised from abdominal wall, agglutination of ileum to injured area (arrow). (D) Specimen for histological examination (view from mucosa of cecum). tum and had a continuous access to fresh water. Animals' welfare was assessed by daily monitoring of weight, behavioral changes, and any mortality with the use of a standard observation chart (body condition scoring, GV-SOLAS, Charité - Universitätsmedizin Berlin).

\section{Surgical Procedure}

General anesthesia was achieved using a weight depending dose of $80 \mathrm{mg} / \mathrm{kg}$ body weight Ketamine and $5 \mathrm{mg} / \mathrm{kg}$ Xylazine ${ }^{\circledR}$. Required level of narcosis for surgery was reached if flexor reflexes failed to appear. The abdomen was then shaved and prepared with alcohol and iodine solution. A $4 \mathrm{~cm}$ median laparotomy was performed to gain access to the abdominal cavity. In the optimized peritoneal adhesion model group (OPAM) $(\mathrm{n}=10)$, the cecum was delivered and kept moist with a watery gauze swab whilst dry gauze was used to gently abrade the cecal peritoneum in a standard manner. Abrasion was repeated until removal of visceral peritoneum, occurring of sub-serosal bleeding, and creation of a homogenous surface of petechial hemorrhages over a $1 \times 2 \mathrm{~cm}$ area. An $1 \times 2 \mathrm{~cm}$ sized patch of parietal peritoneum with the underlying inner muscular layer was sharply resected of the right-lateral abdominal wall (Figure 1A). After replacing the cecum intra-abdominally, both defects were approximated with a $4 / 0$ Prolene $^{\circledR}$ suture to fix the mesentery of the ascending colon to the abdominal wall (Figure 1B). The group without suture fixation $(\mathrm{WSFX})(\mathrm{n}=4)$ represents conventional adhesion models as cecum and abdominal wall, exactly injured as described above, were not approximated. In the sham-OPAM group $(n=5)$ only the approximating suture was placed without peritoneal injuries. In sham-WSFX group $(n=5)$ animals underwent only laparotomy without any injury and/or suturing. The abdomen was closed using two-layer closure technique by a consecutive suture.

\section{Postoperative Management}

After surgery animals were monitored until complete awakening and kept warm with an infrared lamp. Concerning expectable postoperative pain in the immediate postoperative period, animals received novaminsulfone in a body weight adapted dose. All animals were sacrificed on day 7 postoperatively by carbon dioxide narcosis 
followed by cervical disclosure. Immediately, the peritoneal cavity was re-entered via an incision at a left position remote to the original laparotomy scar to prevent disturbing any potentially formed adhesion. Cecum and abdominal wall were harvested for histopathological assessment according to a standard protocol (Figures 1C and 1D).

\section{Evaluation Parameters}

Upon autopsy, cecum and abdominal sidewall were evaluated by macroscopic quantification of adhesion formation by two independent observers according to scoring schemes by Lauder et al. (2011) as well as by Hoffmann et al. (2009) 9,15. The scoring schemes are shown in Table 1 and Table 2. Photographs of the affected areas were also taken for each animal for documentation. The Lauder scoring scheme for measurement of adhesion formation (Table 1) takes into account number, strength, and distribution of adhesions 9. With Hoffmann scoring scheme and grading scale (Table 2) gross adhesions were measured and expressed as a percent of the total de-peritonealized surface area. This was further translated into grades 0-4. Each animal was evaluated for strength of adhesion formation and graded 0-3. The extent of adhesion formation was also graded 0-3. These three sub-scores were summed for a total gross adhesion score ${ }^{15}$. Pathohistological assessment was performed on all cecum and abdominal wall samples to evaluate adhesion formation and the extent of inflammatory response. It was also evaluated, whether there was coverage of damaged tissue by mesothelium/neo-mesothelium. Both, macroscopic and microscopic pathology observers were blinded to the study design and the material used at the time of their evaluation.

\section{Histology}

Samples were excised en bloc, rinsed and immersed in $10 \%$ formaldehyde solution. Specimens were embedded in paraffin blocks. Serial sections were stained with haematoxylin and eosin and alcian-blue and examined by light microscopy to evaluate the structure of the adhesive tissue.

\section{Statistical Analysis}

Statistical analyses were performed with GraphPad PRISM and SSPS software using one-way ANOVA with post-hoc Dunnett's test. All adhesion scores were expressed as the mean (with standard deviation, SD). Different adhesion models were compared by one-way ANOVA with Dunnett's post-test. Differences were considered significant if the probability was less than $0.05(\mathrm{p}<0.05)$.
Table 1: Adhesion scoring scheme by Lauder et al. (2011) 9 .

\begin{tabular}{ll}
\hline Score & Description \\
\hline 0 & No adhesions \\
1 & Thin filmy adhesions \\
2 & More than one thin adhesion \\
3 & Thick adhesion with focal point \\
4 & Thick adhesion with planar attachment \\
5 & Very thick vascularized adhesions or more than one planar
\end{tabular}

Table 2: Adhesion scoring scheme by Hoffmann et al. (2009) ${ }^{15}$.

\begin{tabular}{ll}
\hline Score & Description \\
\hline $\begin{array}{ll}\text { Area Scoring Scheme } \\
0\end{array}$ & No adhesion \\
1 & Cecum to bowel adhesion \\
2 & Cecum to sidewall adhesion over less than $25 \%$ of the \\
3 & Cecum to sidewall adhesion between $25 \%$ and_50\% of \\
4 & Cecum to sidewall adhesion over $50 \%$ of the_surface \\
Strength Scoring Scheme \\
0 & No adhesion \\
1 & Gentle traction required to break adhesion \\
2 & Blunt dissection required to break adhesion \\
3 & Sharp dissection required to break adhesion \\
Extent Scoring Scheme & \\
0 & No adhesion \\
1 & Filmy adhesion \\
2 & Vascularized adhesion \\
3 & Opaque or cohesive adhesion
\end{tabular}

\section{Results}

No animal had to be sacrificed during the course of investigations. All animals showed equitable viability and course of body weight (body weight $\mathrm{d}_{0}$ : $329 \mathrm{~g} \pm 26 \mathrm{~g}, \mathrm{~d}_{7}: 313 \mathrm{~g} \pm 30 \mathrm{~g}$ ). Courses of viability and weight (see Figure 2) were compared statistically to evaluate whether type of surgical intervention had an impact on animal behavior and healing. However, a differentiation according to surgical intervention with respect to these parameters could not be conducted.

Table 3 shows the adhesion scores as classified to the Lauder scoring scheme. The ten animals of the OPAM group had an outstanding adhesion formation response to surgical insult with only one animal having no adhesions at autopsy. For OPAM rats, an average Lauder score of $4.5(\mathrm{SD}=1.6)$ was calculated corresponding with a percentage of $90 \%$ of animals having adhesions. There is a $15 \%$ higher incidence of adhesions in OPAM as compared to WSFX animals as only $75 \%$ of the WSFX rats showed adhesion formation, and of which a range of grades were noted with a mean Lauder score of $3.8(\mathrm{SD}=2.5)$. All animals in both sham-operated control groups did not show any signs of adhesion formation indicating that laparotomy and suture by themselves did not have an impact on adhesion formation in this short-term model. Table 3 further indicates the data that Lauder had experienced with his technique of abrasion and 
enterotomy. The results Lauder obtained with enterotomy were alike in a $90 \%$ range as with OPAM.

Figure 3 compares our data subjected to the Lauder scoring scheme with the original data of the Lauder group. The results obtained by Lauder et al. (2011) with enterotomy correspond with those of OPAM animals while their abrasion of cecum had less impact on adhesion formation ${ }^{9}$.

Figure 4 compares our data subjected to Hoffmann total scores with the original data of Hoffmann group. The impact of adhesion formation in the original data of Hoffmann et al. (2009) is 70\% as compared to OPAM, but $85 \%$ as compared to WSFX ${ }^{15}$.

OPAM was most efficient in provoking adhesions for all compared models $(q>2.1, p<0.01)$ except for the enterotomy method of Lauder et al. (2011) in which the adhesions did not yield significantly different levels of score $(q=0.2, p>0.05)$.

Subjecting the data of our animals to the Hoffmann scoring scheme, a 90\% adhesion formation was observed with a mean total score of $9.0(\mathrm{SD}=3.2)$ in the OPAM group (Table 4). WSFX group had a lower mean total score than OPAM (mean total score $=7.5$, $\mathrm{SD}=5.0$ ). In accordance to the Lauder scoring scheme sham-OPAM and sham-WSFX groups showed no differences between both groups as there were no adhesions.

The histopathological assessment revealed that in all animals with sham-operation the peritoneum was without pathological findings (Figures 5A and 5B) except some minor sub-mucosal inflammatory reaction in the abdominal that might result from the suture (not shown). In the area of agglutination between cecum and abdominal wall, muscular layers of both tissues were melted as can be seen in Figure 5C.

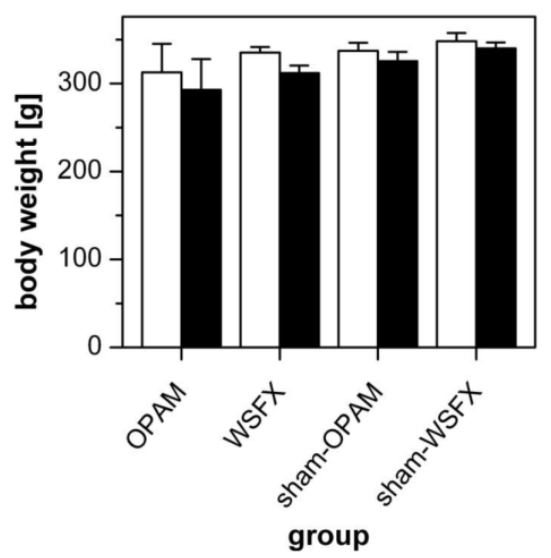

Figure 2: Course of weight analysis. Comparison of $d_{0}$ (white) and $d_{7}$ (black) in all animals of OPAM, WSFX and sham groups showing no significant differences.

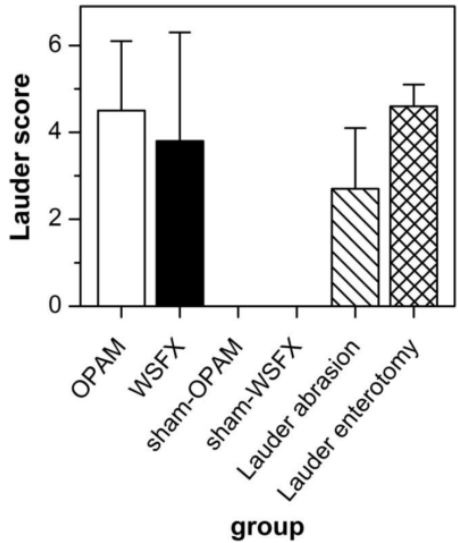

Figure 3: Evaluation using Lauder scoring. OPAM and Lauder enterotomy provide similar efficiency in adhesion formation.

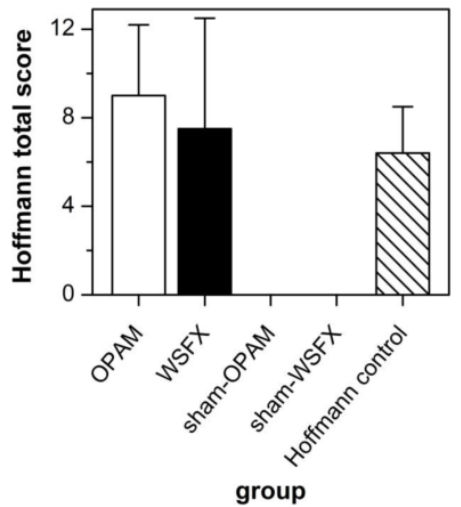

Figure 4: Evaluation using Hoffmann scoring. OPAM provides better efficiency in probability and degree of adhesion vs. conventional models (without suture as well as Hoffmann model).

Table 3: Distribution of Lauder adhesion scores for each group.

\begin{tabular}{|c|c|c|c|c|c|c|c|c|c|c|}
\hline \multirow[t]{2}{*}{ Group } & \multicolumn{10}{|c|}{ Adhesion score } \\
\hline & 0 & 1 & 2 & 3 & 4 & 5 & Total & Mean & SD & Percentage \\
\hline \multicolumn{11}{|l|}{ Experimental results } \\
\hline OPAM & 1 & 0 & 0 & 0 & 0 & 9 & 10 & 4.5 & 1.6 & $90 \%$ \\
\hline WSFX & 1 & 0 & 0 & 0 & 0 & 3 & 4 & 3.8 & 2.5 & $75 \%$ \\
\hline sham-OPAM & 5 & 0 & 0 & 0 & 0 & 0 & 5 & 0.0 & 0.0 & $0 \%$ \\
\hline sham-WSFX & 5 & 0 & 0 & 0 & 0 & 0 & 5 & 0.0 & 0.0 & $0 \%$ \\
\hline \multicolumn{11}{|l|}{ Literature data } \\
\hline Lauder abrasiona & 1 & 3 & 2 & 3 & 6 & 0 & 15 & 2.7 & 1.4 & $53 \%$ \\
\hline Lauder enterotomy & 0 & 0 & 0 & 0 & 4 & 7 & 11 & 4.6 & 0.5 & $92 \%$ \\
\hline
\end{tabular}

Table 4: Hoffmann gross adhesion scores (mean \pm SD) for each group.

\begin{tabular}{llllll}
\hline Group & \multicolumn{5}{l}{ Adhesion score } \\
\cline { 2 - 6 } & Area & Strength & Extend & Total & Percentage \\
\hline Experimental results & & & & \\
OPAM & $3,6 \pm 1,3$ & $2,7 \pm 1,0$ & $2,7 \pm 1,0$ & $9,0 \pm 3,2$ & $90 \%$ \\
WSFX & $3,0 \pm 2,0$ & $2,3 \pm 1,5$ & $2,3 \pm 1,4$ & $7,5 \pm 5,0$ & $75 \%$ \\
sham-OPAM & $0,0 \pm 0,0$ & $0,0 \pm 0,0$ & $0,0 \pm 0,0$ & $0,0 \pm 0,0$ & $0 \%$ \\
sham-WSFX & $0,0 \pm 0,0$ & $0,0 \pm 0,0$ & $0,0 \pm 0,0$ & $0,0 \pm 0,0$ & $0 \%$ \\
Literature data & & & & & \\
Hoffmann & $2,5 \pm 1,1$ & $1,8 \pm 0,6$ & $2,1 \pm 0,6$ & $6,4 \pm 2,1$ & $64 \%$ \\
\hline
\end{tabular}

aControl group with $1 \times 2 \mathrm{~cm}$ sharply excised are of parietal peritoneum and gently abraded cecum by scraping with gauze over a $1 \times 2 \mathrm{~cm}$ area 15 . 

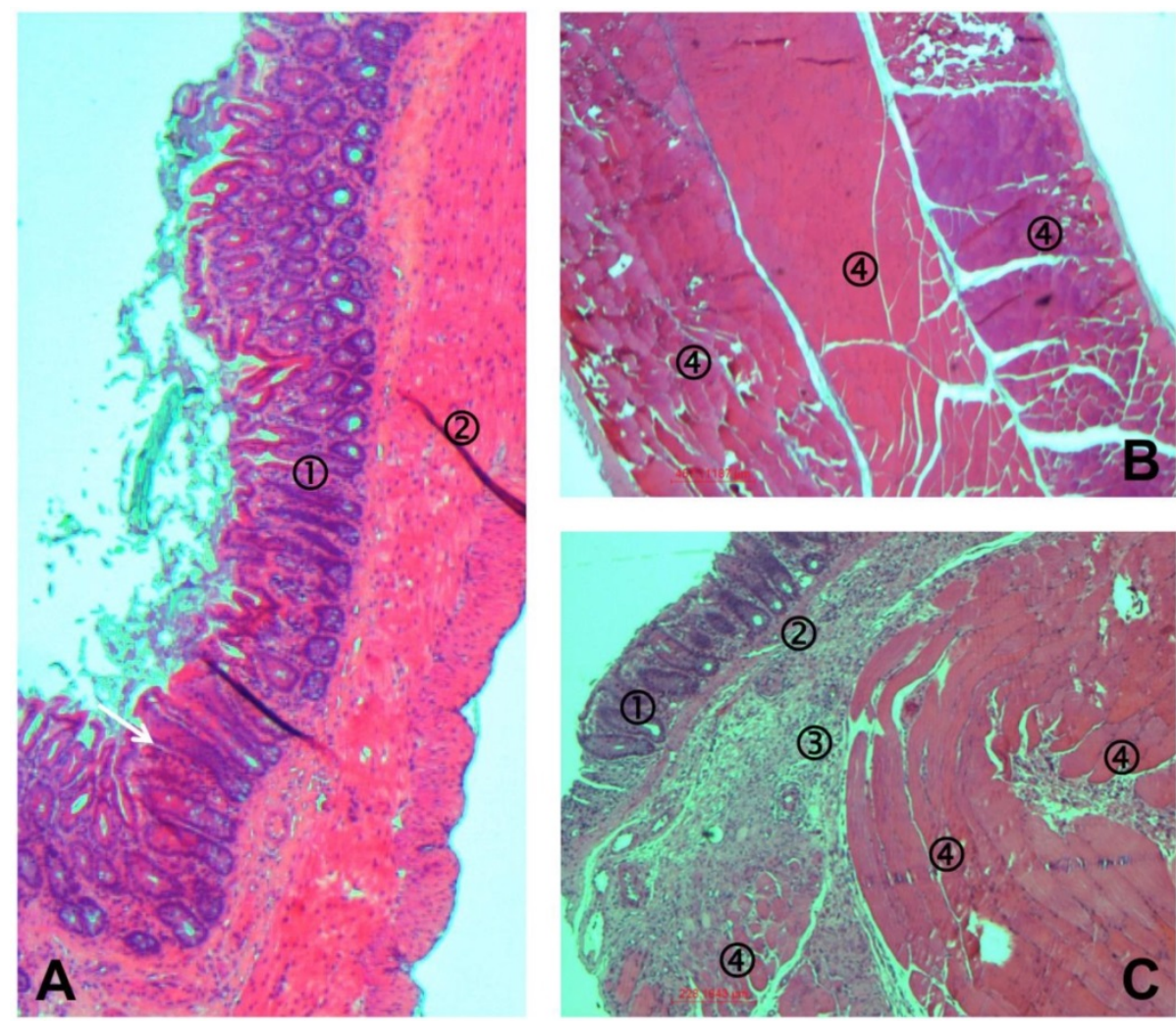

Figure 5: Representative photomicrographs of tissues recovered one week after operation. (A) Cecum of Lewis rat of sham-OPAM group with mucosa (1), smooth muscle (2), and mesothelial coverage. (B) Abdominal wall of Lewis rat of sham-OPAM group with three skeletal muscular layers (4) and mesothelial coverage. (C) Histopathological adhesion in an animal of the OPAM group: transection of the agglutinated area consisting of normal cecal mucosa $\mathbb{1}$, smooth muscle of cecum (2), fibrous tissue (3), and skeletal muscle of abdominal wall (4).

\section{Discussion}

The most frequent cause for abdominal adhesion formation is surgical trauma. Despite a lot of efforts that have been made in improving surgical techniques (e.g. laparoscopic surgery) and development of medical devices for prevention of adhesion formation, there is no satisfactory strategy, yet.

An animal model, which reliably induces adhesion formation under defined conditions, might be a promising basis for development and assessment of new preventive agents and techniques. Rat models represent preferred experimental setting as data of pathological findings in these animals can be transferred well to human conditions.

The term adhesion comprises a variety of pathologies extending from fine fibrous bands to severe non-dissolvable agglutination between organs. A model that induces the latter consistently can be considered as the most strict test condition to determine the quality of adhesion prevention devices.

In their approach to find the "best model" to induce adhesions, Whang et al. (2011) compared parietal peritoneum excision, parietal peritoneum abrasion, peritoneal button creation, and cecal abrasion as trigger measures ${ }^{6}$. The most consistent and repro- ducible results they found was the creation of a peritoneal button using a pestering suture. However, creation of a peritoneal button induces a continuous stimulus for adhesion formation by continuous necrosis over a time span of 5 to 7 days in which physiological peritoneal healing occurs after a defined damage. Thus, this technique does not mimic the common clinical postoperative situation.

The Lauder scoring system is almost identical to further five-point scales 11,13,16-19. Recently Lauder et al. (2011) found an excellent reproducibility (92\% reproducibility) of adhesion formation in rats using a $1 \mathrm{~cm}$ longitudinal incision in the cecum ${ }^{9}$. However, evaluating the photographs of this study, only adhesions appearing as strings were induced. Areas with strict agglutination of organs were missing. Furthermore, a model of enterotomy is not representative for postoperative adhesion formation after an uncomplicated operation.

In 1995 Harris and co-workers used both, creation of a visceral and a parietal lesion to provoke adhesions ${ }^{14}$. The background for this is that peritoneal wounds being opposite implicate the most intense and most reliable stimulus for intense adhesion formation. The paper of Harris is referred to frequently 
because of its close relationship to clinical situations. Recently Hoffmann et al. (2009) added to this technique a scoring system allowing a description of pathology of adhesions in more detail ${ }^{15}$. This scoring allows distinguishing between a wide variety from "no adhesions" to "opaque or cohesive adhesion" (Table 2). The latter - most likely due to the combined injury of abdominal wall defect and cecal abrasion are clinically responsible for the most disgusting complication of adhesions i.e. ileus. Adhesion models like solely abrasion, enterotomy or button technique provoke cohesive adhesions rarely or even not at all and, by this they mimic clinical situation less. Data of their controls showed that even in those, however, inconsistently adhesion formation occurred.

In order to enhance the reproducibility of adhesion formation, an additional suture was placed fixing the mesentery of the ascending colon to the abdominal wall in the here presented OPAM model. By this, the abraded cecum and the abdominal wall defect were approximated. Consequently, macroscopically in all cases adhesions appeared as severe agglutination. This was proven in the histological work-up. Light microscopy showed in the area of adhesion that the smooth muscle layer of the cecum was merged with the skeletal muscle of the abdominal wall. These highest degree adhesions were present in almost all cases proving the excellent reproducibility in our model.

Adhesion creation as performed by using surgical techniques like parietal peritoneum excision and parietal peritoneal abrasion ${ }^{10-13}$ as well as uterine horn ${ }^{12}$ and multiple abrasion models ${ }^{18,20,21}$ generated lower levels of adhesion score in proportion to the grade of our model (data not shown). In contrast, peritoneal bottom creation ${ }^{6}$ yielded equal adhesion grade and quantity compared to or model (data not shown).

In conclusion, our model bases on the model of Harris et al. (1995), which is widely accepted because of its close relation to clinical situation ${ }^{14}$. By additional approximation of injured areas by a suture in the OPAM group, the probability of contact of opposite injured areas was increased. Thus, in almost all animals adhesions could be induced and showed a highest degree of agglutination. The OPAM induces adhesions with excellent probability in incidence and degree of adhesions. Models being as effective as the herein presented OPAM are not suitable for a read out system towards an aggravation of adhesions. However, they are appropriate to evaluate whether products certified for adhesion prevention might be efficient in such severe case scenarios.

\section{Competing Interests}

The authors have declared that no competing interest exists.

\section{References}

1. Hellebrekers BW, Kooistra T. Pathogenesis of postoperative adhesion formation. The British journal of surgery 2011;98:1503-16.

2. Ellis H, Moran BJ, Thompson JN, et al. Adhesion-related hospital readmissions after abdominal and pelvic surgery: a retrospective cohort study. Lancet 1999;353:1476-80.

3. Cates W, Farley TM, Rowe PJ. Worldwide patterns of infertility: is Africa different? Lancet 1985;2:596-8.

4. Trimbos-Kemper TC, Trimbos JB, van Hall EV. Adhesion formation after tubal surgery: results of the eighth-day laparoscopy in 188 patients. Fertility and sterility 1985;43:395-400.

5. Nieuwenhuijzen M, Reijnen MM, Kuijpers JH, van Goor H. Small bowel obstruction after total or subtotal colectomy: a 10-year retrospective review. The British journal of surgery 1998;85:1242-5.

6. Whang SH, Astudillo JA, Sporn E, et al. In search of the best peritoneal adhesion model: comparison of different techniques in a rat model. The Journal of surgical research 2011;167:245-50.

7. Ray NF, Denton WG, Thamer M, Henderson SC, Perry S. Abdominal adhesiolysis: inpatient care and expenditures in the United States in 1994. Journal of the American College of Surgeons 1998;186:1-9.

8. Dijkstra FR, Nieuwenhuijzen M, Reijnen MM, van Goor H. Recent clinical developments in pathophysiology, epidemiology, diagnosis and treatment of intra-abdominal adhesions. Scandinavian journal of gastroenterology Supplement 2000;:52-9.

9. Lauder CI, Garcea G, Strickland A, Maddern GJ. Use of a modified chitosan-dextran gel to prevent peritoneal adhesions in a rat model. The Journal of surgical research 2011;171:877-82.

10. Group TSMS. Prophylaxis of pelvic sidewall adhesions with Gore-Tex surgical membrane: a multicenter clinical investigation. The Surgical Membrane Study Group. Fertility and sterility 1992;57:921-3.

11. Lucas PA, Warejcka DJ, Young HE, Lee BY. Formation of abdominal adhesions is inhibited by antibodies to transforming growth factor-beta1. The Journal of surgical research 1996;65:135-8.

12. Burns JW, Colt MJ, Burgees LS, Skinner KC. Preclinical evaluation of Seprafilm bioresorbable membrane. The European journal of surgery Supplement : = Acta chirurgica Supplement 1997:40-8.

13. Thaler K, Mack JA, Berho M, Grotendorst G, Wexner SD, Abramson SR. Coincidence of connective tissue growth factor expression with fibrosis and angiogenesis in postoperative peritoneal adhesion formation. European surgical research Europaische chirurgische Forschung Recherches chirurgicales europeennes 2005;37:235-41.

14. Harris ES, Morgan RF, Rodeheaver GT. Analysis of the kinetics of peritoneal adhesion formation in the rat and evaluation of potential antiadhesive agents. Surgery 1995;117:663-9.

15. Hoffmann NE, Siddiqui SA, Agarwal S, et al. Choice of hemostatic agent influences adhesion formation in a rat cecal adhesion model. The Journal of surgical research 2009;155:77-81.

16. Becker JM, Dayton MT, Fazio VW, et al. Prevention of postoperative abdominal adhesions by a sodium hyaluronate-based bioresorbable membrane: a prospective, randomized, double-blind multicenter study. Journal of the American College of Surgeons 1996;183:297-306.

17. Oncel M, Remzi FH, Senagore AJ, Connor JT, Fazio VW. Comparison of a novel liquid (Adcon-P) and a sodium hyaluronate and carboxymethylcellulose membrane (Seprafilm) in postsurgical adhesion formation in a murine model. Diseases of the colon and rectum 2003;46:187-91.

18. Oncel M, Remzi FH, Connor J, Fazio VW. Comparison of cecal abrasion and multiple-abrasion models in generating intra-abdominal adhesions for animal studies. Techniques in coloproctology 2005;9:29-33.

19. Thaler K, Mack JA, Zhao RH, et al. Expression of connective tissue growth factor in intra-abdominal adhesions. Diseases of the colon and rectum 2002;45:1510-9.

20. Surrey MW, Friedman S. Second-look laparoscopy after reconstructive pelvic surgery for infertility. The Journal of reproductive medicine 1982;27:658-60.

21. Singer ER, Livesey MA, Barker IK, Hurtig MB, Conlon PD. Development of a laboratory animal model of postoperative small intestinal adhesion formation in the rabbit. Canadian journal of veterinary research $=$ Revue canadienne de recherche veterinaire 1996;60:296-304. 